\title{
A brain marker for developmental speech disorder
}

DOI:

10.1016/j.jpeds.2018.02.043

\section{Document Version}

Accepted author manuscript

Link to publication record in Manchester Research Explorer

\section{Citation for published version (APA):}

Morgan, A. T., Su, M., Reilly, S., Conti-Ramsden, G., Connelly, A., \& Liégeois, F. J. (2018). A brain marker for developmental speech disorder. Journal Of Pediatrics, 198. https://doi.org/10.1016/j.jpeds.2018.02.043

\section{Published in:}

Journal Of Pediatrics

\section{Citing this paper}

Please note that where the full-text provided on Manchester Research Explorer is the Author Accepted Manuscript or Proof version this may differ from the final Published version. If citing, it is advised that you check and use the publisher's definitive version.

\section{General rights}

Copyright and moral rights for the publications made accessible in the Research Explorer are retained by the authors and/or other copyright owners and it is a condition of accessing publications that users recognise and abide by the legal requirements associated with these rights.

\section{Takedown policy}

If you believe that this document breaches copyright please refer to the University of Manchester's Takedown Procedures [http://man.ac.uk/04Y6Bo] or contact uml.scholarlycommunications@manchester.ac.uk providing relevant details, so we can investigate your claim.

\section{OPEN ACCESS}


A brain marker for developmental speech disorder

Short title: A brain marker for developmental speech disorder

Angela T. Morgan*1,2, Merina $\mathrm{Su}^{3}$, Sheena Reilly ${ }^{1,4}$, Gina Conti-Ramsden ${ }^{5}$, Alan Connelly, ${ }^{6}$ Frédérique J. Liégeois ${ }^{3}$

Affiliations

1. Murdoch Children's Research Institute and Royal Children's Hospital, Melbourne, Australia

2. University of Melbourne, Australia

3. UCL Great Ormond Street Institute of Child Health, London, UK

4. Griffith University, Gold Coast, Australia

5. School of Health Sciences, The University of Manchester, UK

6. Florey Institute of Neuroscience and Mental Health, Melbourne, Australia

Corresponding author:

Angela Morgan

Neuroscience of Speech, Murdoch Children's Research Institute, Royal Children's Hospital

Melbourne, Australia. E-mail: angela.morgan@mcri.edu.au; Ph: +61 406971454.

Word count: 2272

\section{STUDY FUNDING}

This study was supported by National Health and Medical Research Council (NHMRC) Centre of Research Excellence (CRE) grant (1023493) awarded to SR, AM and AC; NHMRC CRE (1116976) awarded to AM, AC, SR; NHMRC Career Development Fellowship (607315) and NHMRC Practitioner Fellowship (1105008) awarded to AM; Hearing CRC grant awarded to SR, AM. The authors report no disclosures relevant to the manuscript. Go to Neurology.org for full disclosures. 


\section{ABSTRACT}

Objective: To characterise the organisation of speech- and language-related white matter tracts in children with developmental speech and/or language disorders.

Study design: We collected Diffusion Weighted Imaging (DWI) data from 86 children aged 9 to 11 years, with developmental speech and/or language disorders $(\mathrm{N}=41)$ and typically developing controls (TD, N=45). We used probabilistic tractography of DWI to map language (3 segments of arcuate fasciculus, extreme capsule system) and speech motor (corticobulbar) tracts, bilaterally. The corticospinal and callosal tracts were used as control regions. We compared mean fractional anisotropy (FA) and diffusivity values between atypical and TD groups, co-varying for non-verbal IQ. We then examined differences between atypical sub-groups; developmental speech disorder (DSD), developmental language disorder (DLD), and co-occurring developmental speech and language disorder (DSLD).

Results: FA in the left corticobulbar tract was lower in the DSD than in the TD group. Radial and mean diffusivity were higher in the DSD than the DLD, DSLD or TD groups. There were no group differences for any metrics in the language or control tracts.

Conclusions: Atypical development of the left corticobulbar tract may be a neural marker for DSD. This finding is in line with reports of speech disorder following left corticobulbar damage in children and adults with brain injury. By contrast, we found no association between diffusion metrics in language-related tracts in DLD. Early white matter disruption in DLD may be heterogeneous across cases, in line with the incongruent findings of past MRI studies in the field, and the phenotypic heterogeneity characteristic of this group.

Key words: child; diffusion weighted imaging; tractography; arcuate fasciculus; corticobulbar 


\section{INTRODUCTION}

Developmental speech and language disorders are common, seen in 1 in 20 preschool children, in the absence of neurological deficits, intellectual impairment or hearing loss. ${ }^{1-4}$ These conditions are a leading cause of children presenting to General Practitioners and paediatricians. Developmental speech disorders (DSD; affecting how clearly speech sounds are produced) and language disorders (DLD; affecting language structure such as grammar and semantics) often co-occur, but also dissociate. DLD was previously known as specific language impairment, but international consensus has agreed on DLD nomenclature. ${ }^{2}$ Despite 100 years of phenotypic investigation, no reliable symptom-based prognostic markers exist. Hence interest has intensified in examining MR-based neural markers. Most recently, diffusion weighted imaging and tractography have become promising tools as measures of white matter organization, allowing us to examine structural brain connectivity in these conditions.

A ventral (extreme capsule system) and dorsal route (arcuate fasciculus) have been implicated in typical child language development, with a 'maturational primacy' in the ventral route, present at birth. ${ }^{5,6}$ The dorsal pathway matures at a later stage of development and has been suggested to be involved in more complex language functions. ${ }^{5}$ Few studies, however, have reported reductions in fractional anisotropy (FA) and radial diffusivity (RD) metrics of either stream of this 'traditional language tract' in children with DLD. ${ }^{7-9}$ Of note, the absence of such findings could be in part because existing studies include highly selected, cross-sectionally recruited, clinical samples (see ${ }^{10-12}$ for review), with limited generalizability of findings to the broader DLD population. Current studies also fail to examine 'control' tracts outside hypothesized language regions, obscuring whether findings are localized to language-tracts or widespread throughout the brain. 
White matter integrity has not been examined in DSD to date, yet left corticobulbar tract deficits have been reported in childhood dysarthria after brain injury ${ }^{13}$, childhood stuttering ${ }^{14}$, adult dysarthria ${ }^{15}$ and even in an adult case of neurodegenerative speech disorder in the absence of language disorder. ${ }^{16}$ Further, in relation to the developing system, no study has directly compared white matter integrity of speech and language disordered groups together, limiting knowledge of shared or distinct neural underpinnings.

Here we examined white matter connectivity in children with DLD, DSD and typically developing controls. Participants were recruited from a longitudinal community cohort study of speech and language. We hypothesized developmental speech and language disorders would be associated with atypical development of speech-motor (corticobulbar) and language (dorsal and ventral streams) tracts, respectively.

\section{METHODS}

\section{Participant recruitment}

Participants ( $\mathrm{N}=86$, age range: 9.25-11.25 years) were recruited from the Early Language in Victoria Study (ELVS), a longitudinal community-based study of 1900 children. $^{3}$

Communication status was collected almost annually from 8- 10 months of age $\mathrm{e}^{3}$ up to the age of the current study (i.e., 9 to 11 years). Age of scanning was carefully chosen to reflect a time when communication trajectories are relatively stable. ${ }^{17}$ Ethical approval was granted by the Royal Children's Hospital Human Research Ethics Committee (Reference number HREC31225).

Participants were recruited to 4 groups based on longitudinal data: Typically developing controls (TD, n=45); Developmental Language Disorder (DLD, n=13); Developmental 
Speech disorder (DSD, n=17); and co-occurring Developmental Speech and Language disorders (DSLD, $\mathrm{n}=11$ ). The three latter groups were combined into an "atypical" group for the first stage of analyses; followed by sub-group analyses.

Inclusion criteria for all 4 groups included non-verbal IQ $\geq 80$ on the Kaufman Brief Intelligence Test ${ }^{18}$ administered at age 4 and the Wechsler Abbreviated Scales of Intelligence $(\text { WASI-II })^{19}$ at age 7 . DLD and TD groups were required to have typical speech as assessed via the Goldman Fristoe Test of Articulation $2^{\text {nd }}$ Edition $\left(\right.$ GFTA-II) ${ }^{20}$ at ages 4 and 7 . The DLD group was also required to have impaired language, defined as a total language score of $\leq 81(1.25 \mathrm{SD} \text { below the mean })^{3}$ on the CELF-IV at two time points between at ages 4 and 7 , or 5 and 7. Some attrition occurred across the 4 and 5 year old waves of the ELVS, hence expanding the DLD inclusion criteria across 2 data waves provided a larger pool for recruitment. In contrast, children with DSD had articulation or phonological speech errors consistent with DSD at ages 4 and $7 .{ }^{21}$ To assess speech performance, the Goldman-Fristoe Test of Articulation; GFTA-II (Goldman and Fristoe, 2005) was administered as a singleword test that elicits all the speech sounds of English in initial, medial and final positions. All sounds were transcribed and assessed for the presence of articulation and phonological errors to confirm a diagnosis of DSD. ${ }^{21,22}$ Articulation disorder was denoted as phonetic-based distortions (e.g., lisps, de-rhoticism) where occurrence of the distortion was more frequent than correct production of that phone. Articulation disorder could also include an omission error where the phone was absent in the child's inventory, but it appears in the phonetic inventory of $>90 \%$ of peers in normative data. ${ }^{22-24}$ Phonological delay was use of a phonological process that occurs in typically developing speech, but is used beyond an age where it is typically resolved in $>90 \%$ of peers ${ }^{23}$ Phonological disorder was use of a phonological process that is atypical and seen in $<10 \%$ the normative sample population at 
any age. ${ }^{23}$ Conversational samples were also rated to confirm the presence of errors noted in single-word stimuli in connected speech to provide further evidence of persistence. In addition, DSD and TD groups were required to have normal language scores $(\geq 85)$ as per the Clinical Evaluation of Language Fundamentals - preschool $^{25}$ at age 4, and Clinical Evaluation of Language Fundamentals $4^{\text {th }}$ Edition $(\mathrm{CELF}-\mathrm{IV})^{26}$ at ages 5 and 7 . Exclusion criteria were a history of neurological, hearing, genetic or neurodevelopmental disorders (e.g., autistic spectrum disorder) and non-English speaking background.

\section{Procedure}

\section{Clinical assessment at time of scanning}

At the time of scanning, participants were assessed with the same speech, language and nonverbal IQ tests reported above for participant group selection (Table 1). Standardized scores were used for the CELF-IV ${ }^{26}$ and WASI. ${ }^{19}$ Clinical diagnosis of DSD was made based on phonetic transcription and phonological process analysis. ${ }^{21,22}$

\section{MRI acquisition}

T1-weighted images were acquired with an isotropic resolution of $0.9 \mathrm{~mm}$ (inversion time = 900 milliseconds, repetition time $=1,900$ milliseconds, echo time $=2.6$ milliseconds, flip angle $=9^{\circ}$, matrix size $256 \times 256,160$ partitions) on a 3-Tesla Tim Trio MRI scanner (Siemens Medical Solutions, Erlangen, Germany). A conventional T2 scan was also acquired and the $\mathrm{T} 1$ and $\mathrm{T} 2$ scans were reviewed by a clinical radiologist. A diffusion-weighted dataset of 64 directions using a twice-refocused, spin-echo, echo planar imaging sequence was also acquired with: $\mathrm{b}$ value $=3,000 \mathrm{~s} / \mathrm{mm} 2$, echo time/repetition time $=110 / 8,300$ milliseconds, field of view $=240 \times 240 \mathrm{~mm}$, matrix size $=96 \times 96$, slice thickness $=2.5 \mathrm{~mm}$ 
(isotropic voxel size $=2.5 \times 2.5 \times 2.5 \mathrm{~mm}$ ), 60 contiguous axial slices (total acquisition $\approx 9$ minutes).

\section{Preprocessing of MRI datasets}

DWI datasets were pre-processed using MRtrix.${ }^{27}$ Fractional anisotropy (FA) and eigenvector maps were extracted. Constrained spherical deconvolution was used to estimate the distribution of fiber orientations. ${ }^{28,29}$ This is an optimal method relative to the diffusion tensor model, in areas with multiple crossing fibers. ${ }^{30,31}$

\section{Tractography}

A single rater (M.S.), blinded to participants' status, performed tractography using MRtrix. ${ }^{28}$ Tracking was performed using a probabilistic streamlines algorithm ${ }^{31}$ from spherical seed regions (7-mm radius) to generate 3 segments of arcuate fasciculus (AF) ${ }^{32}$, the corticobulbar tract (CBT, as in Liegeois et al. $\left.{ }^{13}\right)$, and the extreme capsule (ExC) system bilaterally (see Figure 1 for seed and inclusion regions). To assess the specificity of our findings to speech and language regions, we also delineated and measured diffusion values from white matter tracts not hypothesized to be related to speech or language, namely the corticospinal tract (CST) originating from the hand motor region ${ }^{13}$ and the whole corpus callosum. The maximum number of streamlines generated was set at 10,000 and we retained a maximum of 1,000 streamlines passing through both the seed and inclusion regions. Masks were generated to encompass voxels with a minimum of 40 streamlines for each tract. Mean FA and diffusion values were averaged across all voxels contained in this binary mask.

\section{Statistical analysis of MRI-derived data}


The 13 tract values were compared between TD and 3 combined atypical (DS/LD) groups using multivariate analysis of covariance (NVIQ as covariate) in the Statistical Package for the Social Sciences 21.0 for Windows. ${ }^{33}$ A mixed model analyses of covariance (ANCOVAs) was then performed for each tract (Hemisphere x Group design), with NVIQ as covariate. Where group differences were detected, we examined subgroup differences (DSD, DLD, DSLD) using post hoc t-tests. Non-parametric methods (Mann-Whitney tests) were used for confirmation where data was not normally distributed. The $\alpha$ level was set at 0.05 (2-tailed) and adjusted (Bonferroni) to correct for multiple comparisons when performing post hoc analyses.

\section{RESULTS}

\section{Clinical presentation}

The 4 groups were matched for demographic characteristics (Table 1). Male:female ratios were comparable across groups (DSD, 7:10; DSLD, 6:5; DLD, 7:6, TD: 21:24). Non-verbal IQ scores, although within the typical range, were lower in DLD children than in children with DSD as commonly reported in this group. ${ }^{17}$ As expected, children with DLD had significantly lower CELF-IV scores (total, receptive, and expressive) than the other two groups, whereas children with DSD had significantly lower GFTA-II scores. Children with DSD and DSLD presented with articulation disorder, phonological disorder and phonological delay (Figure 2; online). Children with DSLD had a more phonological presentation at age 4 but by age 9-11, at time of scanning, the majority of this group also had articulation errors. Slightly more participants in the DSD (72\%) than DSLD (64\%) group had articulation errors at time of scanning. Children with DLD and DSLD had mixed profiles of expressive and 
receptive language impairments (Table 1) and mixed profiles across the domains of semantics, syntax and morphology.

\section{Tractography}

Conventional MRI scans were confirmed to be normal. Tractography delineated all tracts in all participants, with the exception of 8 instances in which no streamlines were generated for the posterior segment of the $\mathrm{AF}(\mathrm{TD}=4, \mathrm{DLD}=1, \mathrm{DSD}=3, \mathrm{DSLD}=3)$. In these instances, we treated tractography-derived measures as missing values. We included the other 74 track values in the analyses.

MANCOVA revealed no overall significant difference between the TD and atypical group (combined DSD, DLD, DSLD groups; $\mathrm{F}=1.12, p=.360$ ), but a significant difference for the left CBT only $(\mathrm{F}=5.36, p=.024 ; p>0.18$ in all other cases), see Figure 3. Consistent with this finding, univariate analyses also revealed trends for a group difference and group by hemisphere effect for the CBT only. When all four groups were entered in the same model in a one-way ANCOVA, main effect of group was confirmed for FA in the left CBT $(\mathrm{F}=2.73$, $p=.049$ ) and non significant for the right CBT. Post-hoc tests showed that FA in the left CBT was significantly different between DSD and TD groups only (Bonferroni corrected $p=.045$ ). The Shapiro-Wilks test indicated that data were not normally distributed in $\operatorname{TD}(p<0.001$, $d f=45$ ) or the DSLD groups ( $p=.043, d f=41)$, but non-parametric tests confirmed that FA in the left CBT was significantly lower in the DSD relative to the TD group ( $\mathrm{U}=213, p=.007$; and $p>0.05$ when compared to other groups). Of note, no additional group differences were detected when NVIQ was not used as a covariate. 
We further examined FA differences found in the left corticobulbar tract. MANCOVA of $\mathrm{MD}, \mathrm{RD}$, and $\mathrm{AD}$ metrics for the left $\mathrm{CBT}$ in the atypical group compared to TD, with nonverbal IQ as a co-variate, revealed a significant group difference in $\mathrm{RD}(\mathrm{F}=3.97, p=.050)$, with the atypical group having higher RD. There were no significant differences in AD $(\mathrm{p}>.27)$ or MD of this tract $(p>0.12)$ (Table 2; online). Examining subgroup differences, univariate ANCOVAs with all four groups revealed an overall effect of group for both RD $(\mathrm{F}=4.74, p=.004)$ and $\mathrm{MD}(\mathrm{F}=3.98, p=.01)$ but not $\mathrm{AD}(\mathrm{F}=1.27, p=.29)$ for the left $\mathrm{CBT}$ (Table 3; online). Post hoc comparisons revealed the DSD group had higher values than both the TD (RD: $p=0.01 ; \mathrm{MD}: p=.007)$ and DLD groups (RD: $p=.03$; MD: $p=.004$ ).

\section{DISCUSSION}

We report the first association between developmental speech disorder and reduced FA of the left corticobulbar tract, suggesting atypical development of this tract may be a neural marker for DSD.

Altered connectivity of the left CBT has previously been associated with speech disorder in different childhood populations and disease models including childhood stuttering ${ }^{14}$ and dysarthria after childhood traumatic brain injury. ${ }^{13}$ In adult cases, left hemisphere lesions to regions along the CBT as tracked here (i.e., ventral to hand omega), have also been associated with acute dysathria. ${ }^{15,34-36}$ It seems there is converging evidence suggesting the left corticobulbar tract is associated with speech execution in children and adults across a range of disease mechanisms, and even in typical development.

By contrast, we did not find a significant association between classical language tracts and DLD. Evidence is scarce in this field, with one study supporting an association between 
reduced fractional anisotropy in both dorsal and ventral routes ${ }^{8}$ and another study, in the SLF alone. ${ }^{7}$ A further study reported increased mean diffusivity in children with DLD relative to controls, but did not mention differential effects of fractional anisotropy across groups. ${ }^{9}$ Interestingly, one study has reported core deficits in the corpus callosum, rather than traditional language tracts, in cases with DLD. ${ }^{37}$

Lack of consistency of findings across studies may be due to methodological differences including the application of other MR metrics in developmental speech and language disorders. ${ }^{10-12}$ Methodological approaches unique to this study represented important methodological strengths in the study of potential neural markers. These included longitudinally-informed selection of participants from a community cohort, within a narrow age range, and co-varying for NVIQ. We carefully matched both typical and atypical groups from the same cohort with longitudinal phenotyping and typical communication status of the TD group confirmed from 8-10 months; enabling more robust comparisons than previous studies in this field. We also minimized issues related to the influence of crossing fibers on tractography by using an algorithm robust to crossing fibers. ${ }^{29}$ Further, that we did identify a significant difference in the DSD group implies our study was sufficiently powered to detect an effect of this size.

Another possible explanation for why an association between DLD (as well as the subgroup of children with both DSLD) and language pathways was not detected, is that white matter disruption may be highly heterogeneous in children with DLD, in line with the incongruence of findings from past MRI studies in this field, ${ }^{10-12}$ and the recognised phenotypic heterogeneity of this group, ${ }^{1-4}$ including in our sample. Similarly we did not detect changes in the left corticobulbar tract for the group with co-occurring speech and language difficulties, i.e., the DSLD group. This finding could be due to the fact that a slightly greater 
Paper accepted by Journal of Pediatrics February 15th, 2018

percentage of the DSD than DSLD group had a motor-based articulation disorder at both the 4 and 7 year old assessment time points, which may have increased our power to detect white matter changes in the motor tract of this group.

Clinically, to our knowledge, we provide the first data suggesting alterations to the left corticobulbar tract is likely to be a marker of developmental speech disorder. Further longitudinal study would be required to determine whether this measure could be a sensitive and specific prognostic tool. 


\section{AUTHOR CONTRIBUTIONS}

AM, FL, SR, AC, GCR designed the research, supervised the data collection. AM, SR, AC wrote the grant application and obtained grant funding to conduct the research. LP collected clinical and MR data. AM, FL, MS, AC, SR, GCR performed the research. MS, FL analyzed and interpreted the MRI data. AM, FL, LP, MS, SR, GCR analysed and interpreted the behavioural data. All authors contributed to writing and revision of the final manuscript.

\section{ACKNOWLEDGMENTS}

The authors thank all participants and their families for their time and support. The authors also thank J.-D.T. for helpful input regarding MRTrix, Lauren Pigdon (Murdoch Children's Research Institute, Melbourne; nil relevant funding sources and nil conflicts of interest) for data collection and Shawna Farquharson (Florey Institute of Neuroscience and Mental Health Melbourne; nil relevant funding sources and nil conflicts of interest) and her team of radiographers for scanning participants. AM, SR, and AC are grateful to the Operational Infrastructure Support Program of the State Government of Victoria for their support. 


\section{REFERENCES}

1. Bishop DV, Snowling MJ, Thompson PA, Greenhalgh T; CATALISE consortium. CATALISE: A Multinational and Multidisciplinary Delphi Consensus Study. Identifying Language Impairments in Children. PLoS One 2016; 11(7):e0158753.

2. Bishop DV, Snowling MJ, Thompson PA, Greenhalgh T; CATALISE-2 consortium. Phase 2 of CATALISE: a multinational and multidisciplinary Delphi consensus study of problems with language development: Terminology. J Child Psychol Psychiatry 2017 Epub Mar 30. doi: 10.1111/jcpp.12721.

3. Reilly S, Wake M, Ukoumunne OC, Bavin E, Prior M, Cini E, et al. Predicting language outcomes at 4 years of age: findings from Early Language in Victoria Study. Pediatrics. 2010; 126: e1530-7.

4. Eadie P, Morgan A, Ukoumunne OC, Ttofari Eecen K, Wake M, Reilly S. Speech sound disorder at 4 years: prevalence, comorbidities, and predictors in a community cohort of children. Dev Med Child Neurol 2015;57:578-84.

5. Brauer J, Anwander A, Perani D, Friederici AD. Dorsal and ventral pathways in language development. Brain Lang 2013;127:289-95.

6. Skeide MA, Friederici AD. The ontogeny of the cortical language network. Nature Reviews Neuroscience 2016;17:323-332.

7. Verhoeven JS, Rommel N, Prodi E, Leemans A, Zink I, Vandewalle E et al. Is there a 
Paper accepted by Journal of Pediatrics February 15th, 2018

common neuroanatomical substrate between autism spectrum disorder and specific language impairment? Cereb Cortex, 2012; 22: 2263-2271.

8. Vydrova R, Komarek V, Sanda J, Sterbova K, Jahodova A, Maulisova A et al. Structural alterations of the language connectome in children with specific language impairment. Brain Lang 2015;151:35-41.

9. Roberts TP, Heiken K, Zarnow D, Dell J, Nagae L, Blaskey L et al. Left hemisphere diffusivity of the arcuate fasciculus: influences of autism spectrum disorder and language impairment. Am J Neuroradiol 2014;35:587-92.

10. Liégeois F, Mayes A, Morgan A. Neural Correlates of Developmental Speech and Language Disorders: Evidence from Neuroimaging. Curr Dev Disord Rep 2014;7;1:215227.

11. Mayes AK, Reilly S, Morgan AT. Neural correlates of childhood language disorder: a systematic review. Dev Med Child Neurol 2015; 57:706-17.

12. Morgan A, Bonthrone A, Liégeois FJ. Brain basis of childhood speech and language disorders: are we closer to clinically meaningful MRI markers? Curr Opin Pediatr 2016;28:725-730.

13. Liégeois F, Tournier JD, Pigdon L, Connelly A, Morgan AT. Corticobulbar tract changes as predictors of dysarthria in childhood brain injury. Neurology. 2013; 80:926-32.

14. Connally EL, Ward D, Howell P, Watkins KE. Disrupted white matter in language and motor tracts in developmental stuttering. Brain Lang 2014;131:25-35.

15. Urban PP, Rolke R, Wicht S, Keilmann A, Stoeter P, Hopf HC et al. Left-hemispheric dominance for articulation: prospective study on acute ischaemic dysarthria at different localizations. Brain 2006;129:767-777. 
16. Silveri MC, Incordino F, Lo Monaco R, Bizzarro A, Masullo C, Piludu F et al. Neural substrates of the 'low-level' system for speech articulation: Evidence from primary opercular syndrome. J Neuropsychol 2016 E-pub ahead of print Feb 7.

17. Conti-Ramsden G, St Clair MC, Pickles A, Durkin K. Developmental trajectories of verbal and nonverbal skills in individuals with a history of specific language impairment: from childhood to adolescence. J Speech Lang Hear Res 2012;55:1716-35.

18. Kaufman AS, Kaufman, NL. Kaufman Brief Intelligence Test. $2^{\text {nd }}$ Ed. American Guidance Services; Circle Pines, MN: 2004.

19. Wechsler D, Hsiao-pin C. Wechsler Abbreviated Scale of Intelligence (2nd ed.) (WASIII). Pearson, Bloomingdale, MN: 2011.

20. Goldman R, Fristoe M. Goldman-Fristoe Test of Articulation (2nd ed.) (GFTA-2). Pearson, MN: 2000.

21. Morgan A, Ttofari Eecen K, Pezic A, Brommeyer K, Mei C, Eadie P et al. Who to Refer for Speech Therapy at 4 Years of Age Versus Who to "Watch and Wait"? J Pediatr 2017.

22. Dodd B, Ttoffari-Eecen T, Brommeyer K, Reilly S, Morgan A. (2017). Delayed and Disordered Development of Articulation and Phonology between Four and Seven Years. Child Language Teaching and Therapy.

23. Dodd B, Zhu H, Crosbie C, Holm A, Hua Z, Ozanne A. Diagnostic Evaluation of Articulation and Phonology. Pearson Australia.

24. Dodd B, Holm A, Hua Z, Crosbie S. Phonological development: a normative study of British English-speaking children. Clin Linguist Phon 2003;17:617-643.

25. Wiig EH, Secord WA, Semel E. Clinical Evaluation of Language Fundamentals Preschool (2nd ed.) (CELF-P2). Pearson, MN: 2006.

26. Semel E, Wiig EH, Secord WA. Clinical Evaluation of Language Fundamentals - (4th ed.) (CELF-4). Pearson, MN: 2006. 
27. J.-D. Tournier, Brain Research Institute, Melbourne, Australia, http://www.brain.org.au/software/

28. Tournier JD, Calamante F, Gadian DG, Connelly A. Direct estimation of the fiber orientation density function from diffusion-weighted MRI data using spherical deconvolution. Neuroimage 2004;23:1176-1185.

29. Tournier JD, Calamante F, Connelly A. Robust determination of the fibre orientation distribution in diffusion MRI: non-negativity constrained super-resolved spherical deconvolution. Neuroimage 2007;35:1459-1472.

30. Tournier JD, Yeh CH, Calamante F, Cho KH, Connelly A, Lin CP. Resolving crossing fibres using constrained spherical deconvolution: validation using diffusion-weighted imaging phantom data. Neuroimage 2008;42:617-625.

31. Tournier JD, Calamante F, Connelly A. MRtrix: Diffusion tractography in crossing fiber regions. Imaging Syst Tech 2012;22:53-66.

32. Catani M. From hodology to function. Brain 2007;130:602-5.

33. Statistical Package for the Social Sciences (SPSS) 21.0 for Windows, Chicago, IL.

34. Kim JS, Kwon SU, Lee TG. Pure dysarthria due to small cortical stroke. Neurology 2003;60:1178-1180.

35. Urban PP, Hopf HC, Fleischer S, Zorowka PG, Müller- Forell W. Impaired cortico-bulbar tract function in dysarthria due to hemispheric stroke: functional testing using transcranial magnetic stimulation. Brain 1997;120:1077-1084.

36. Terao Y, Ugawa Y, Yamamoto T, Sakurai Y, Masumoto T, Abe O et al. Primary face motor area as the motor representation of articulation. J Neurol 2007;254:442-447.

37. Kim J, Kim YW, Park CI, Park ES, Kim HH, Lee SK et al. Diffusion-tensor magnetic resonance imaging in children with language impairment. NeuroReport 2006;17:12791282. 
Paper accepted by Journal of Pediatrics February 15th, 2018 
Paper accepted by Journal of Pediatrics February 15th, 2018

Figure 1. Seed and inclusion regions of interest (ROIs) used for tractography with corresponding reconstructed tracts

T1/orientation

Tract

Seed regions

Anterior segment: seed ROI $1+$ exclusion ROI2

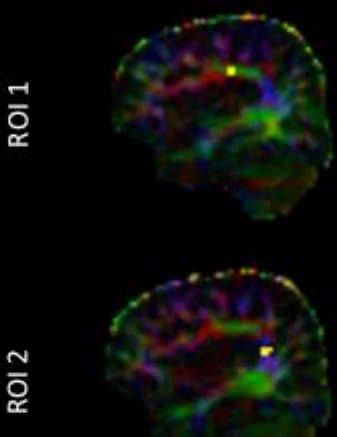

Direct segment: seed ROI $1+$

inclusion ROI2

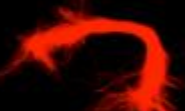

ס̄

Posterior segment: seed ROI $2+$ inclusion ROI3 - exclusion ROI 1
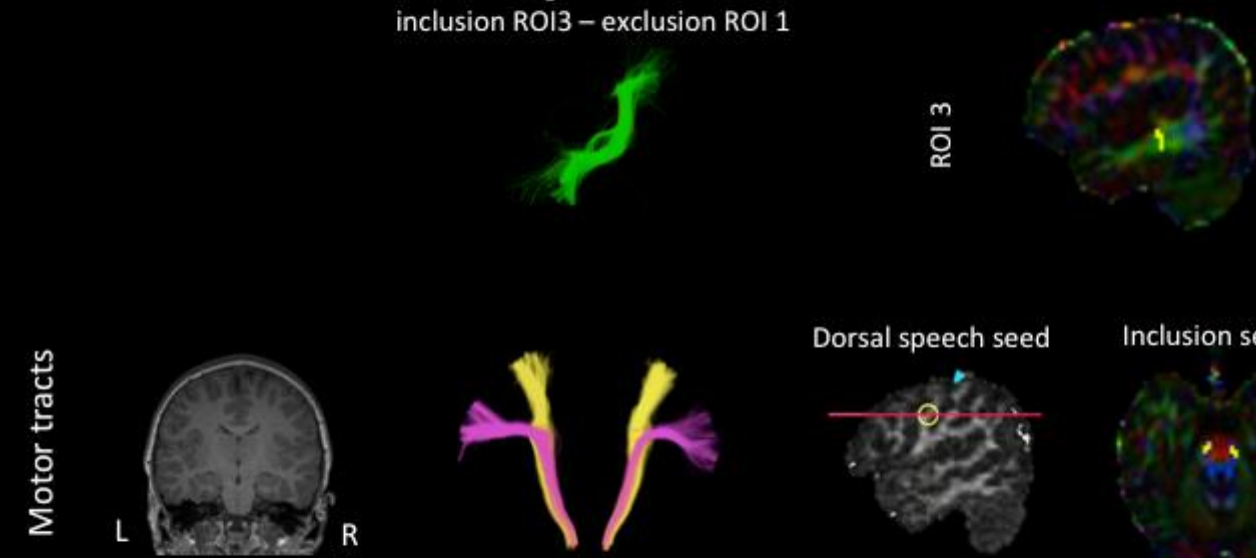

Dorsal speech seed

Inclusion seed
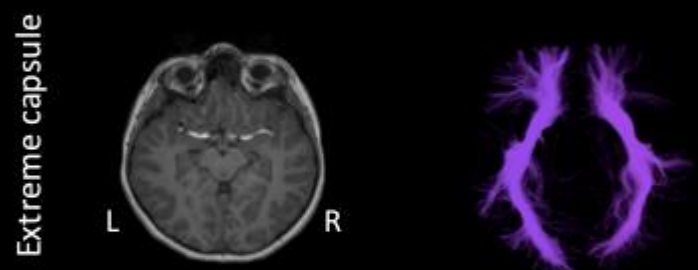

Dorsal view

Coronal view
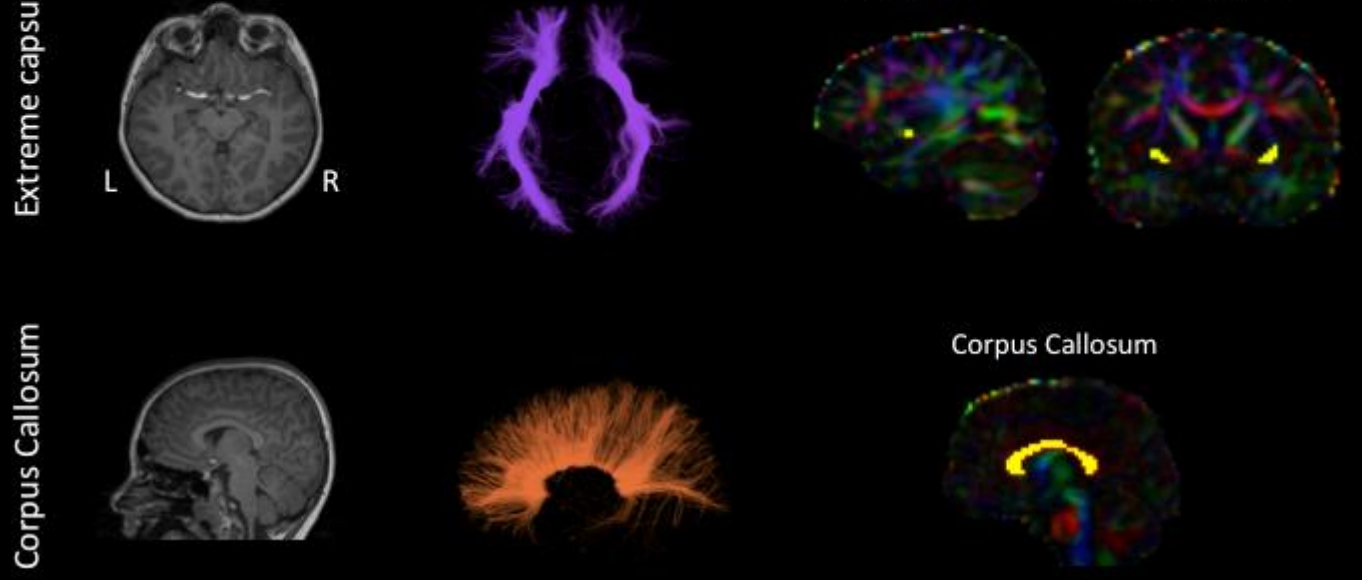

Corpus Callosum
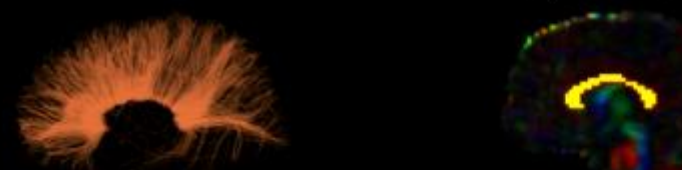
Paper accepted by Journal of Pediatrics February 15th, 2018

Figure 2 - online only. Speech error types at age 4 years and age 9-11 years for DSD (N=17) and DSLD (N=11) groups

A) Speech error types for DSD group at 4 years

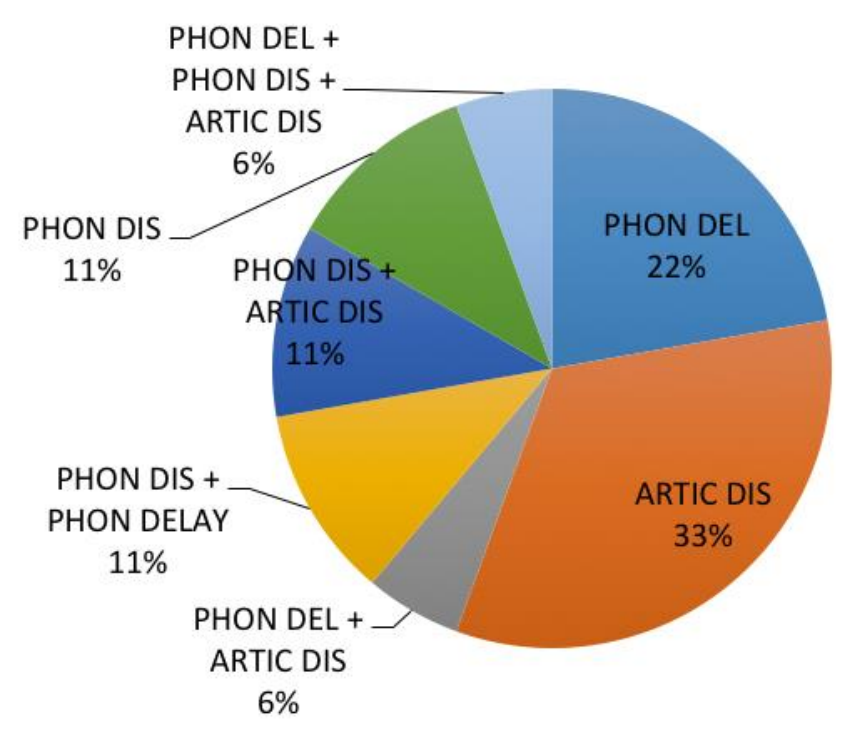

C) Speech error types for DSLD group at 4 years

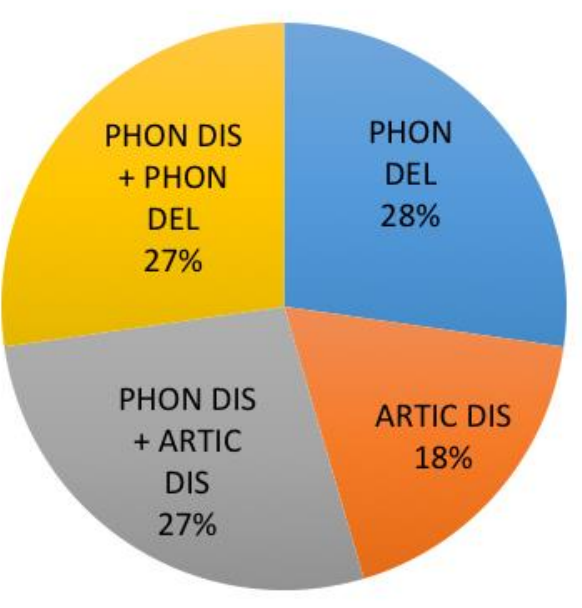

NB: \% denotes proportion of cases with specific diagnosis
B) Speech error types for DSD group at 9-11 years

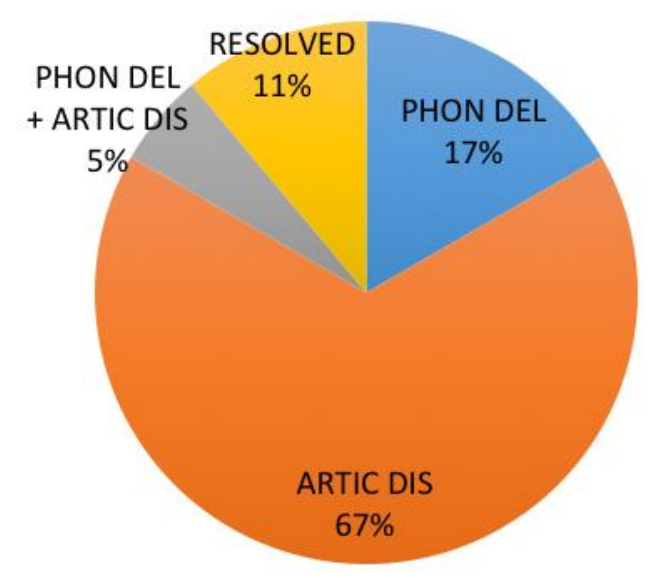

D) Speech error types for DSLD group at 9-11 years

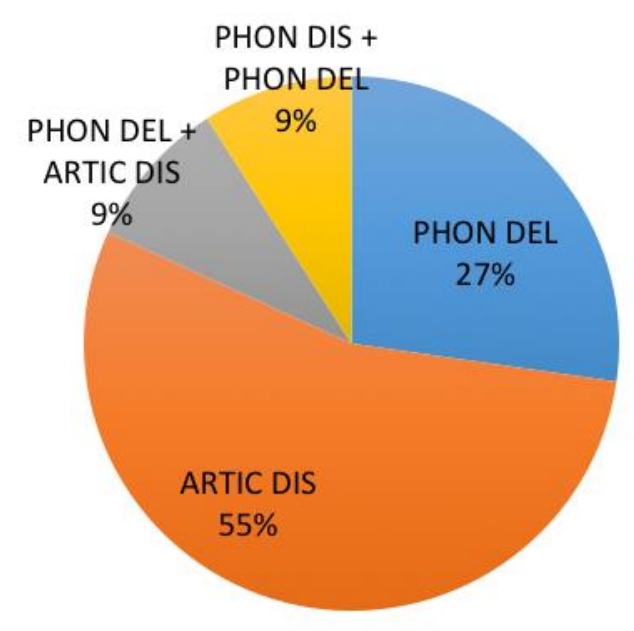


Paper accepted by Journal of Pediatrics February 15th, 2018

Figure 3. Fractional anisotropy mean and standard deviation for typical (TD group) and atypical groups combined (DSD, DLD, DSLD groups).

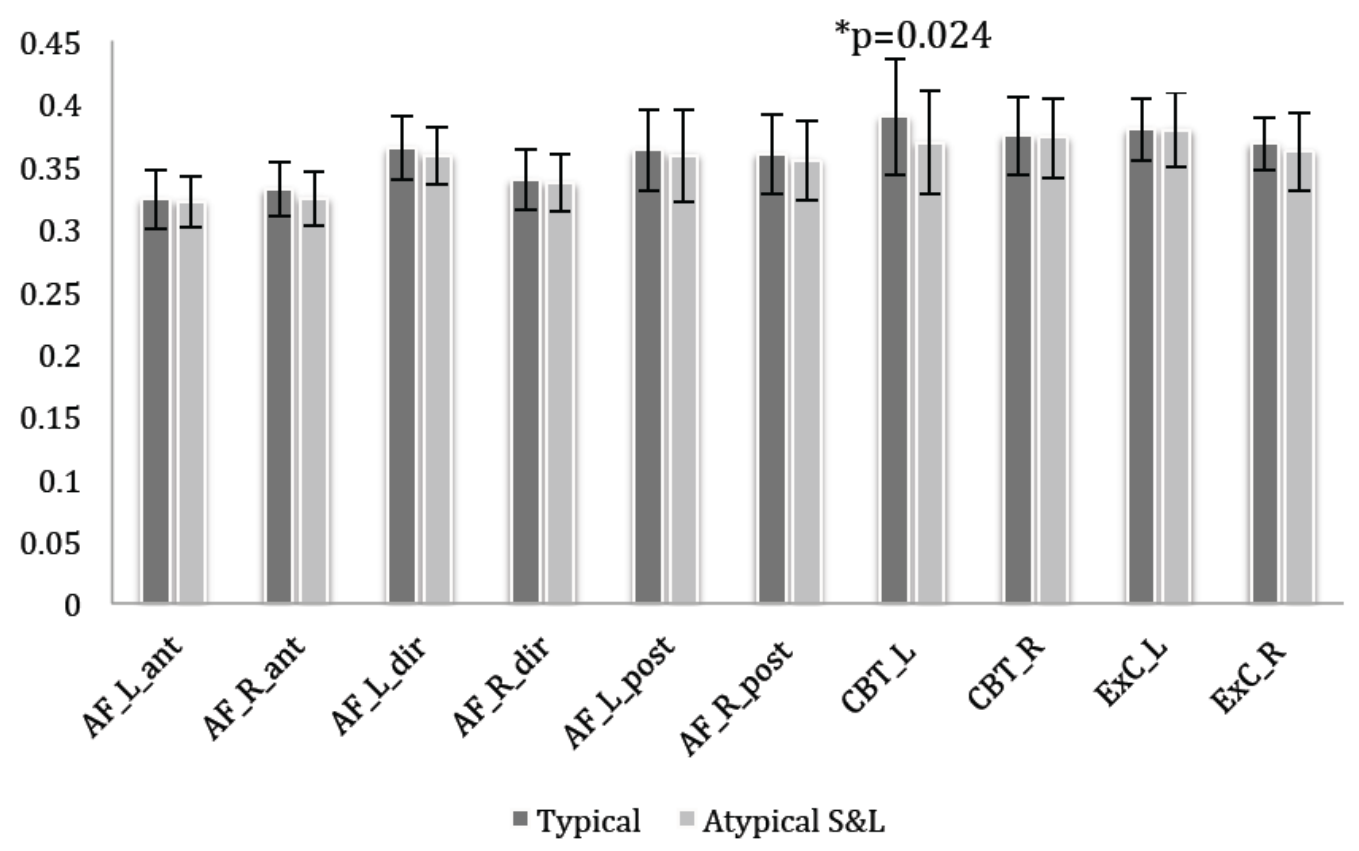


Paper accepted by Journal of Pediatrics February 15th, 2018

Figure 3 Legend

AF_L_ant: anterior arcuate fasciculus left hemisphere; $A F \_R \_a n t:$ anterior arcuate fasciculus right hemisphere; $\mathrm{AF} \_$L_dir: direct arcuate fasciculus left hemisphere; AF_R_dir: direct arcuate fasciculus right hemisphere; AF_L_post: posterior arcuate fasciculus left hemisphere; AF_R_post: posterior arcuate fasciculus right hemisphere; CBT_L: left corticobulbar tract; CBT_R: right corticobulbar tract; ExC_L: left extreme capsule; ExC_R: right extreme capsule. 
Paper accepted by Journal of Pediatrics February 15th, 2018

\section{Table 1}

Sample characteristics for typically developing (TD), developmental speech disorder (DSD), developmental language disorder (DLD), developmental speech and language disorder (DSLD) groups

\begin{tabular}{|c|c|c|c|c|c|c|c|c|}
\hline & \multirow[b]{2}{*}{$\mathrm{TD}(n=45)$} & \multirow[b]{2}{*}{$\operatorname{DSD}(n=17)$} & \multirow{2}{*}{$\begin{array}{c}\text { Group } \\
\text { DLD }(n=13)\end{array}$} & \multicolumn{3}{|c|}{ Test statistics } & \multirow{2}{*}{\multicolumn{2}{|c|}{$\begin{array}{l}\text { Effect size } \\
\eta^{2}\end{array}$}} \\
\hline & & & & $\operatorname{DSLD}(n=11)$ & Df & Statistic & & \\
\hline Age at testing (months) & $123.47 \pm 6.59$ & $123.06 \pm 3.51$ & $123.23 \pm 2.68$ & $125.18 \pm 3.52$ & 3 & $H=1.55$ & .671 & .02 \\
\hline Non-verbal IQ & $102.09 \pm 9.76$ & $106.06 \pm 11.22$ & $94.46 \pm 9.71$ & $96.91 \pm 10.09$ & 3 & $H=11.13$ & .011 & .10 \\
\hline CELF-4 Core Language & $107.20 \pm 8.78$ & $102.88 \pm 8.45$ & $85.31 \pm 9.32$ & $83.27 \pm 10.71$ & 3 & $F=33.99$ & $<.001$ & .55 \\
\hline CELF-4 Receptive & $105.40 \pm 8.74$ & $102.12 \pm 6.69$ & $86.46 \pm 7.63$ & $84.45 \pm 14.62$ & 3 & $H=38.90$ & $<.001$ & .44 \\
\hline CELF-4 Expressive & $109.02 \pm 9.76$ & $104.12 \pm 9.30$ & $87.77 \pm 10.89$ & $83.09 \pm 11.47$ & 3 & $F=29.07$ & $<.001$ & .52 \\
\hline GFTA-2 & $103.29 \pm 2.06$ & $99.59 \pm 4.49$ & $103.15 \pm 2.15$ & $100.27 \pm 4.73$ & 3 & $H=12.89$ & .005 & .12 \\
\hline
\end{tabular}

$n=$ sample size; $\mathrm{Df}=$ Degrees of freedom. Values are means \pm SD. 
Paper accepted by Journal of Pediatrics February 15th, 2018

Table 2 - online only. Post-hoc analyses of mean diffusivity (MD), radial diffusivity (RD) and axial diffusivity (AD) in left corticobulbar (left CBT) tract for atypical combined (DSD, DLD, DSLD) and typically developing (TD) groups.

\begin{tabular}{lllll}
\hline Measure & TD & $\begin{array}{l}\text { Atypical } \\
\text { combined }\end{array}$ & F & $p$ \\
MD left CBT & 0.0005589 & $\begin{array}{l}0.0005673 \\
(0.000022)\end{array}$ & 2.44 & .122 \\
RD left CBT & $\begin{array}{l}0.0004376 \\
(0.000025)\end{array}$ & $\begin{array}{l}0.0004530 \\
(0.000035)\end{array}$ & 3.97 & .050 \\
AD left CBT & $0.0008015)$ & $\begin{array}{l}0.0007960 \\
(0.000024)\end{array}$ & 1.21 & .274 \\
& $(0.000027)$ & $(0.025)$ & \\
\hline
\end{tabular}

Mean (SD) 
Paper accepted by Journal of Pediatrics February 15th, 2018

Table 3 - online only. Summary of comparisons for mean diffusivity (MD), radial diffusivity (RD) and axial diffusivity (AD) in left corticobulbar (left CBT) tract across the 4 groups (TD, DSD, DLD, DSLD)

\begin{tabular}{lllllll}
\hline Measure & TD & DSD & DLD & DSLD & F & $p$ \\
MD & 0.0005589 & 0.0005786 & 0.0005555 & 0.0005656 & 4.74 & .004 \\
& $(0.000022)$ & $(0.000024)$ & $(0.000025)$ & $(0.000019)$ & & \\
RD & 0.0004376 & 0.0004673 & 0.0004376 & 0.0004514 & 3.98 & .011 \\
& $(0.000035)$ & $(0.000032)$ & $(0.000037)$ & $(0.000026)$ & & \\
AD & 0.0008015 & 0.0008012 & 0.0007913 & 0.0007939 & 1.27 & .290 \\
& $(0.000027)$ & $(0.000021)$ & $(0.000025)$ & $(0.000029)$ & & \\
\hline
\end{tabular}

Mean (SD) 